\title{
Efficacy and safety of tranexamic acid in unilateral major revision total hip arthroplasty
}

\author{
Lin Mei, Hongxing Li, Weihong Zhu, Yong Luo, Xinzhan Mao \\ Department of Orthopedics, The Second Xiangya Hospital of Central South University, Changsha, China \\ Contributions: (I) Conception and design: L Mei, H Li, X Mao; (II) Administrative support: X Mao; (III) Provision of study materials or patients: \\ W Zhu, X Mao; (IV) Collection and assembly of data: HX Li, Y Luo; (V) Data analysis and interpretation: L Mei, H Li, Y Luo; (VI) Manuscript \\ writing: All authors; (VII) Final approval of manuscript: All authors. \\ Correspondence to: Xinzhan Mao. Department of Orthopedics, The Second Xiangya Hospital of Central South University, Changsha, China. \\ Email: xinzhan.mao@csu.edu.cn.
}

Background: The risk of blood loss differs among subtypes of revision total hip arthroplasty (THA), and different tranexamic acid (TXA) protocols have rarely been studied in those conditions. The present study aimed to evaluate the efficacy and safety of intravenous and intravenous plus topical TXA in a subtype of revision THA.

Methods: We retrospectively reviewed 91 patients who underwent unilateral major revision THA from 2010 to 2018. The major revision was defined as a subtype of revision THA, which included concomitant femoral and acetabular components revision, revision for periprosthetic femoral fracture (PFF), and one-stage revision for periprosthetic joint infection (PJI). In the intravenous group, 23 patients received intravenously $1 \mathrm{~g}$ of TXA 30 minutes before the incision with the second dose 3 hours later. In the combined group, 20 patients received intravenously $1 \mathrm{~g}$ of TXA 30 minutes before the incision with the second dose 3 hours later, and $2 \mathrm{~g}$ of TXA was topically injected around the joint capsule when the fascia layer was closed. Forty-eight patients who underwent revision procedures without TXA constituted the control group. Within the three groups, we compared demographic variables, operation-related data, transfusion volume, transfusion rate, calculated blood loss, postoperative drainage volume, and venous thromboembolism (VTE) risk.

Results: Compared with the control group, both intravenous and combined TXA significantly reduced intraoperative transfusion volume $(3.43 \pm 2.32$ vs. $4.68 \pm 2.63$ units, $\mathrm{P}=0.044 ; 2.78 \pm 1.91$ vs. $4.68 \pm 2.63$ units, $\mathrm{P}=0.004$; respectively) and total transfusion volume (4.16 \pm 2.73 vs. $5.73 \pm 3.05$ units, $\mathrm{P}=0.036 ; 3.50 \pm 2.74$ vs. $5.73 \pm 3.05$ units, $\mathrm{P}=0.005$; respectively), and there were significant reductions of postoperative drainage volume $(250.87 \pm 204.54$ vs. $455.73 \pm 303.93 \mathrm{~mL}, \mathrm{P}=0.003 ; 285.00 \pm 218.14$ vs. $455.73 \pm 303.93 \mathrm{~mL}, \mathrm{P}=0.017$; respectively) and calculated blood loss $(1,322.49 \pm 656.13$ vs. $1,698.66 \pm 728.39 \mathrm{~mL}, \mathrm{P}=0.031 ; 1,237.13 \pm 545.32$ vs. $1,698.66 \pm 728.39 \mathrm{~mL}, \mathrm{P}=0.012$; respectively). One patient had a symptomatic pulmonary embolism, and two patients had calf muscular vein thrombosis in the control group. There were two patients and one patient with calf muscular vein thrombosis in the intravenous group and the combined group, respectively. Perioperative transfusion volume, transfusion rate, and calculated blood loss were comparable between the intravenous group and the combined group.

Conclusions: Both intravenous TXA and combined TXA significantly reduced perioperative transfusion volume and calculated blood loss in unilateral major revision THA with comparable perioperative transfusion rate and risk of VTE. More researches are required to explore the optimal TXA administration protocol in subtypes of revision THA.

Keywords: Blood loss; venous thromboembolism (VTE); tranexamic acid (TXA); transfusion; revision total hip arthroplasty (THA) 
Submitted Oct 14, 2019. Accepted for publication Jul 28, 2020.

doi: 10.21037/apm-19-372

View this article at: http://dx.doi.org/10.21037/apm-19-372

\section{Introduction}

Tranexamic acid (TXA) reduces bleeding and transfusion in primary total hip arthroplasty (THA) without increasing the risk of venous thromboembolism (VTE) (1-6). Compared with primary THA, revision THA usually causes more perioperative blood loss and transfusion because of more extensive exposure, longer operation time, and osteotomy.

Several studies have shown that intravenous TXA reduced perioperative blood loss $(7,8)$ and transfusion (8-12) without increasing the risk of VTE $(7,9,11,12)$ in revision THA. Compared with intravenous TXA, intravenous plus topical TXA resulted in significantly less perioperative blood loss and transfusion with a comparable VTE risk in revision THA (13). Nevertheless, given the variable surgical indications and the complexity of revision THA, studies $(8,13-15)$ have shown that risk of perioperative blood loss significantly differed among subtypes of revision THA, such as isolated femoral component revision, isolated acetabular component revision, and concomitant femoral and acetabular components revision, indicating the heterogeneity of revision THA. However, the efficacy and safety of different TXA protocols have hardly been evaluated in subtypes of revision THA.

The present study aimed to evaluate the efficacy and safety of intravenous TXA and intravenous plus topical TXA and compare the two protocols in a subtype of revision THA.

We present the following article in accordance with the STROBE reporting checklist (available at http://dx.doi. org/10.21037/apm-19-372).

\section{Methods}

\section{Study design and patients}

We retrospectively reviewed 91 patients who underwent unilateral major revision THA from January 2010 to December 2018 in our hospital. We defined major revision as a subtype of revision THA, which included concomitant femoral and acetabular components revision, revision for periprosthetic femoral fracture (PFF), and one-stage revision for periprosthetic joint infection (PJI). Patients who underwent unilateral major revision THA were included in this study. Patients with the following situations were excluded: explantation procedures or re-implantation procedures in two-stage revision for PJI, allergy to TXA (Liduoxin $^{\circledR}$, Haiwang Pharmaceutical, China), history of VTE, malignant tumor, hemorrhagic diseases, preoperative anticoagulant use, and unavailable data. Patients who underwent contralateral primary THA during unilateral revision THA were also excluded.

It was not until October 2014 that TXA was clinically used in our hospital. Patients who underwent unilateral major revision THA without TXA were defined as the control group. Patients who received intravenous TXA or intravenous plus topical TXA constituted the intravenous TXA group or the combined TXA group, respectively.

Revision procedures were performed by one of four senior surgeons. A posterolateral approach with general anesthesia was used in all cases. Drainage was routinely applied.

This study was conducted in accordance with the Declaration of Helsinki (as revised in 2013) and was approved by the Ethics Committee of the Second Xiangya Hospital of Central South University (2019-192). Informed consent was waived because of the nature of a retrospective study. The patient's personal data have been secured.

\section{TXA protocols and VTE prophylaxis}

In the intravenous group, $1 \mathrm{~g}$ of TXA was administered intravenously 30 minutes prior to incision, followed by a second dose 3 hours later. In the combined group, $1 \mathrm{~g}$ of TXA was administered intravenously 30 minutes prior to incision, followed by a second dose 3 hours later, and $2 \mathrm{~g}$ of TXA was injected topically around joint capsule when fascia layer was closed.

Inpatients received low molecular weight heparin (LMWH) (Qizheng ${ }^{\circledR}$, Qilu Pharmaceutical, China) 5,000 IU daily or rivaroxaban (Xarelto ${ }^{\circledR}$, Bayer AG, Germany) $10 \mathrm{mg}$ daily. The physical prophylaxis, such as ankle pump, contraction of quadriceps, and early ambulation, was also 
used to decrease the risk of VTE events.

\section{Data collection and calculation}

This study retrieved data from the medical records of patients. Demographics included age, gender, height, weight, American Society of Anesthesiologists (ASA) score, and comorbidity. Preoperative data included surgical indications, preoperative hemoglobin, preoperative hematocrit, international normalized ratio (INR), and D-dimer. Intraoperative data included the surgical approach, operation time, types of revision component, and intraoperative allogenic blood transfusion volume. Postoperative data included the lowest postoperative hemoglobin and hematocrit, postoperative drainage volume, postoperative allogenic blood transfusion volume, VTE prophylactic protocols, and in-hospital VTE events.

Calculated blood loss was calculated as described by Huerfano (16). The Intraoperative or postoperative transfusion rate was calculated by dividing the number of patients receiving intraoperative or postoperative allogenic blood transfusion by the total number of patients in the relevant group, respectively. The overall transfusion rate was calculated by dividing the number of patients receiving either intraoperative or postoperative allogenic blood transfusion by the total number of patients in the relevant group.

\section{Statistical analysis}

All continuous data were presented as mean \pm standard deviation. The one-way analysis of variance test was used to compare the difference of continuous data if a normal distribution and equal variances were present, such as age, body mass index (BMI), hemoglobin, hematocrit, international normalized ratio (INR), D-dimer, operation time, calculated blood loss, intraoperative and total transfusion volume, and postoperative drainage volume. The Kruskal-Wallis test was applied for continuous data without a normal distribution, such as postoperative transfusion volume. The Fisher's exact test was used for nominal scaled variables. A pairwise comparison test was conducted if there was a significant difference in the oneway analysis of variance test, Kruskal-Wallis test, or Fisher's exact test. All statistical tests were performed by SPSS 20.0 software and considered significant if a $\mathrm{P}$ value $<0.05$.

\section{Results}

From January 2010 to December 2018, a total of 145 patients underwent revision THA in our institution. Among these patients, one patient underwent isolated femoral head exchange, and three and thirteen patients underwent isolated femoral revision and isolated acetabular revision, respectively. Eighteen patients underwent implant explantation procedures for PJI, and nine patients received re-implantation procedures in two-stage revision for PJI. One patient underwent bilateral revision THA, and three patients underwent contralateral primary THA during revision surgeries. There were preoperative VTE events in five patients. One patient with thrombocytopenia was also excluded. The remaining 91 patients were enrolled in this study. There were 48, 23, and 20 patients in the control group, the intravenous group, and the combined group, respectively.

No significant differences were observed among the three groups in terms of gender, age, BMI, ASA score, surgical indication, comorbidity, preoperative hemoglobin, preoperative hematocrit, INR, and D-dimer (Table 1). The most common surgical indication was aseptic pain, and there were 46 (95.8\%), 21 (91.3\%), and 17 (85.0\%) patients with aseptic pain in the control group, the intravenous group, and the combined group, respectively. In the combined group, two patients (10.0\%) underwent one-stage revision THA for PJI. All remaining patients in the three groups underwent unilateral major revision THA for PFF.

There were no significant differences in terms of the operation time, types of revision component, and VTE prophylactic protocols among the three groups (Table 2). The operation time was the longest in the control group (250.50 \pm 86.99 minutes), which is comparable with the intravenous group $(213.43 \pm 44.35$ minutes $)$ and the combined group (231.45 \pm 80.04 minutes). The cemented component was the most common revision prosthesis, with $32(66.7 \%), 13(56.5 \%)$, and $15(75.0 \%)$ patients in the control group, the intravenous group, and the combined group, respectively. LMWH or rivaroxaban was routinely used for VTE prophylaxis. A total of 32 (66.7\%), 16 $(69.6 \%)$, and $10(50.0 \%)$ patients received $\mathrm{LMWH}$, and $16(33.3 \%), 7(30.4 \%)$, and $10(50.0 \%)$ patients received 
Table 1 Demographics and preoperative data of unilateral major revision THA

\begin{tabular}{|c|c|c|c|c|}
\hline Variables & Control group & Intravenous group & Combined group & $P$ value \\
\hline Gender, male, n (\%) & 19 (39.6) & $12(52.2)$ & $7(35.0)$ & $0.519^{\star \star}$ \\
\hline Age (years) & $62.92 \pm 8.99$ & $66.52 \pm 10.57$ & $68.45 \pm 12.65$ & $0.100^{*}$ \\
\hline $\operatorname{BMl}\left(\mathrm{kg} / \mathrm{m}^{2}\right)$ & $22.84 \pm 2.48$ & $22.52 \pm 2.74$ & $22.03 \pm 2.59$ & $0.499^{*}$ \\
\hline II & $26(54.2)$ & $10(43.5)$ & $7(35.0)$ & \\
\hline III & $22(45.8)$ & $10(43.5)$ & $11(55.0)$ & \\
\hline IV & 0 & $3(13.0)$ & $2(10.0)$ & \\
\hline Surgical indications, n (\%) & & & & $0.119^{* *}$ \\
\hline PJI & 0 & 0 & 2 & \\
\hline Hypertension, n (\%) & $20(41.7)$ & $13(56.5)$ & $10(50.0)$ & $0.504^{\star *}$ \\
\hline Diabetes, n (\%) & 7 (14.6) & $2(8.7)$ & 0 & $0.206^{\star \star}$ \\
\hline CAD, n (\%) & $7(14.6)$ & $1(4.3)$ & $3(15.0)$ & $0.442^{* \star}$ \\
\hline Hemoglobin (g/L) & $126.02 \pm 13.76$ & $127.48 \pm 15.41$ & $123.75 \pm 17.86$ & $0.721^{*}$ \\
\hline Hematocrit (\%) & $37.95 \pm 4.24$ & $39.21 \pm 3.94$ & $38.48 \pm 4.96$ & $0.518^{\star}$ \\
\hline INR & $0.97 \pm 0.12$ & $0.96 \pm 0.12$ & $0.97 \pm 0.08$ & $0.923^{*}$ \\
\hline D-dimer ( $\mu \mathrm{g} / \mathrm{mL})$ & $1.41 \pm 1.11$ & $0.96 \pm 0.80$ & $1.32 \pm 0.81$ & $0.199^{*}$ \\
\hline
\end{tabular}

Values were given as the mean \pm standard deviation for the continuous data and the number with the percentages for the nominal scaled variables. *, one-way analysis of variance; ${ }^{\star \star}$, Fisher's exact test. THA, total hip arthroplasty; BMI, body mass index; ASA score, American Society of Anesthesiologists score; PFF, periprosthetic femoral fracture; PJI, periprosthetic joint infection; CAD, coronary artery disease; INR, international normalized ratio.

rivaroxaban in the control group, the intravenous group, and the combined group, respectively.

Compared with the control group, both intravenous TXA and intravenous plus topical TXA significantly reduced intraoperative transfusion volume $(3.43 \pm 2.32$ vs. $4.68 \pm 2.63$ units, $\mathrm{P}=0.044 ; 2.78 \pm 1.91$ vs. $4.68 \pm 2.63$ units, $\mathrm{P}=0.004$; respectively) and total transfusion volume (4.16 \pm 2.73 vs. $5.73 \pm 3.05$ units, $\mathrm{P}=0.036 ; 3.50 \pm 2.74$ vs. $5.73 \pm 3.05$ units, $\mathrm{P}=0.005$; respectively) (Table 3). The intraoperative transfusion rate was significantly lower in the intravenous group compared with the control group ( $80.0 \%$ vs. $97.9 \%, \mathrm{P}<0.05$ ), while no significant differences were observed in postoperative transfusion rate and overall transfusion rate among the three groups.

Compared with the control group, both intravenous TXA and intravenous plus topical TXA significantly decreased calculated blood loss $(1,322.49 \pm 656.13$ vs. $1,698.66 \pm 728.39 \mathrm{~mL}, \mathrm{P}=0.031 ; 1,237.13 \pm 545.32$ vs. $1,698.66 \pm 728.39 \mathrm{~mL}, \mathrm{P}=0.012$; respectively) and postoperative drainage volume $(250.87 \pm 204.54 \mathrm{vs}$. $455.73 \pm 303.93 \mathrm{~mL}, \mathrm{P}=0.003 ; 285.00 \pm 218.14$ vs. $455.73 \pm 303.93 \mathrm{~mL}, \mathrm{P}=0.017$; respectively) (Table 4). Because of the limited population and relatively high overall transfusion rate in the present study, we compared the lowest postoperative hematocrit and hemoglobin instead of the drop of hematocrit and hemoglobin. The lowest postoperative hematocrit was significant higher in the intravenous group and the combined group compared with the control group $(28.11 \% \pm 4.49 \%$ vs. $24.60 \% \pm 3.66 \%$, $\mathrm{P}=0.002 ; 27.24 \% \pm 5.21 \%$ vs. $24.60 \% \pm 3.66 \%, \mathrm{P}=0.022$; respectively). The lowest postoperative hemoglobin was significantly higher in the intravenous group compared 
Table 2 Intraoperative data and VTE prophylaxis of unilateral major revision THA

\begin{tabular}{llll}
\hline Variables & Control group & Intravenous group & Combined group \\
\hline Patients, $\mathrm{n}$ & 48 & 23 & 20 \\
$\begin{array}{l}\text { Operation time (minutes) } \\
\text { Revision component, } \mathrm{n}(\%)\end{array}$ & $250.50 \pm 86.99$ & $213.43 \pm 44.35$ & $231.45 \pm 80.04$ \\
Cemented & & & \\
Cementless & $32(66.7)$ & $13(56.5)$ & $15(75.0)$ \\
Hybrid & $8(16.7)$ & $7(30.4)$ & $2(10.0)$ \\
VTE prophylaxis, $\mathrm{n}(\%)$ & $8(16.6)$ & $3(13.1)$ & $3(15.0)$ \\
LMWH & $32(66.7)$ & $16(69.6)$ & $10(50.0)$ \\
Rivaroxaban & $16(33.3)$ & $7(30.4)$ & $10(50.0)$ \\
\hline
\end{tabular}

Values were given as the mean \pm standard deviation for the continuous data and the number with the percentages for the nominal scaled variables. *, one-way analysis of variance; **, Fisher's exact test. VTE, venous thromboembolism; THA, total hip arthroplasty; LMWH, low molecular weight heparin.

Table 3 Transfusion rate and volume in unilateral major revision THA

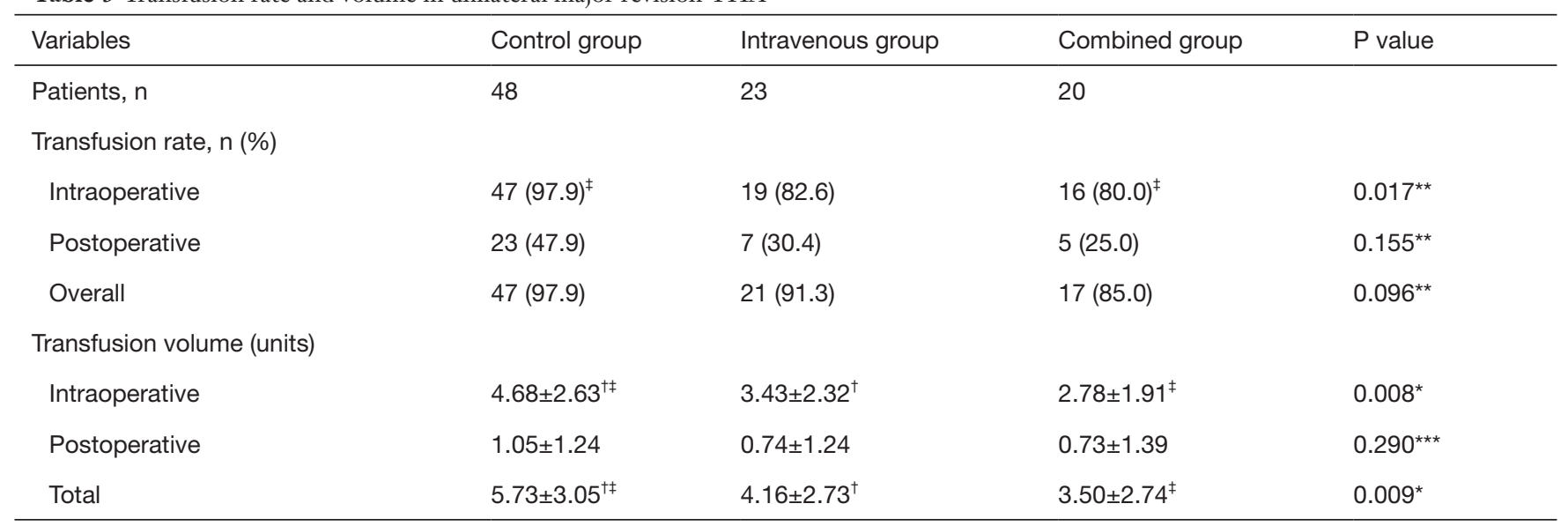

Values were given as the mean \pm standard deviation for the continuous data and the number with the percentages for the nominal scaled variables. *, one-way analysis of variance; ${ }^{\star \star}$, Fisher's exact test; ${ }^{\star * *}$, Kruskal-Wallis test; ${ }^{\dagger, \neq}$, a pairwise comparison showed significant difference $(\mathrm{P}<0.05)$. THA, total hip arthroplasty.

with the control group $(92.35 \pm 15.04$ vs. $82.88 \pm 12.05 \mathrm{~g} / \mathrm{L}$, $\mathrm{P}=0.008$ ) (Table 4).

Moreover, we found no significant differences in perioperative transfusion rate and perioperative transfusion volume between the intravenous group and the combined group (Tables 3,4).

Notably, the VTE event rate was not significantly different among the three groups (Table 4), indicating that neither intravenous TXA nor intravenous plus topical TXA increased the risk of VTE. Specifically, one patient had a symptomatic pulmonary embolism, and two patients had calf muscular vein thrombosis in the control group. There were two patients and one patient with postoperative calf muscular vein thrombosis in the intravenous group and the combined group, respectively.

\section{Discussion}

Both protocols of TXA significantly reduced calculated blood loss and perioperative transfusion volume with comparable perioperative transfusion rate and the risk of VTE in unilateral major revision THA. Although previous 
Table 4 Calculated blood loss and postoperative data in unilateral major revision THA

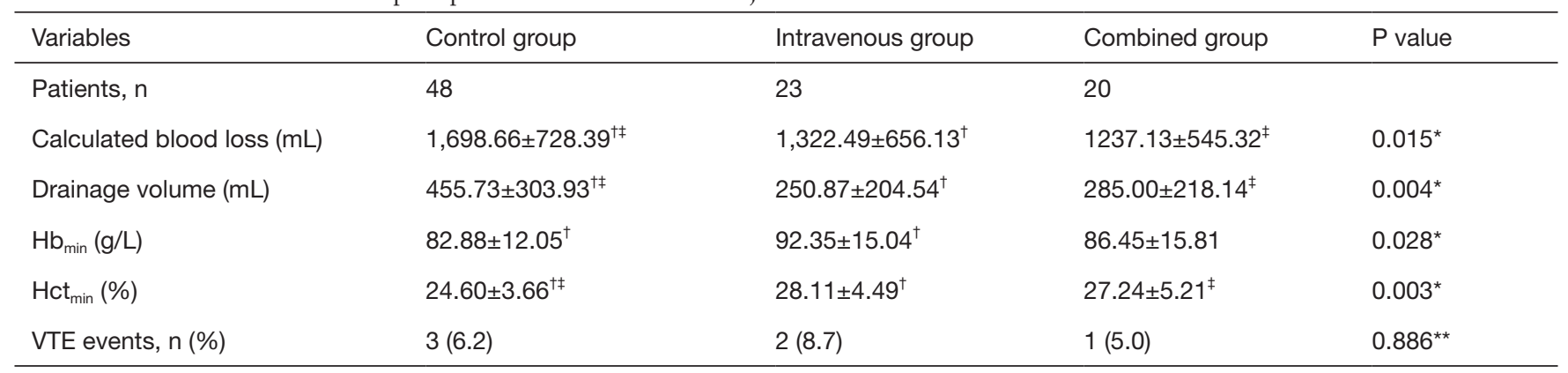

Values were given as the mean \pm standard deviation for the continuous data and the number with the percentages for nominal scaled variables. *, One-way analysis of variance; ${ }^{* *}$, Fisher's exact test; ${ }^{\dagger},{ }^{\ddagger}$, a pairwise comparison showed significant difference $(\mathrm{P}<0.05)$. THA, total hip arthroplasty; $\mathrm{Hb}_{\min }$, the minimum postoperative hemoglobin; $\mathrm{Hct}_{\text {min, }}$, the minimum postoperative hematocrit; VTE, venous thromboembolism.

studies (7-12) showed that intravenous TXA reduced blood loss and transfusion in revision THA without increasing VTE risk, the majority of these studies did not consider the heterogeneity of subtypes of revision THA. Zarin et al. (15) demonstrated that revision THA involving both acetabular and femoral components had significantly more total blood loss than isolated femoral component revision and isolated acetabular component revision $(1,603 \pm 739$ vs. $1,258 \pm 597 \mathrm{~mL}, \mathrm{P}=0.02 ; 1,603 \pm 739$ vs. $1,114 \pm 530 \mathrm{~mL}$, $\mathrm{P}=0.0001$; respectively), whereas isolated femoral and acetabular component revision had similar total blood loss. Peck et al. (8) confirmed that revision THA, involving deep infection, PFF, and both femoral and acetabular components, had significantly more estimated blood loss compared with isolated femoral or acetabular component revision. However, Garvin et al. (14) reported that total blood loss was similar between femoral revision and revision of both components $(1,078 \pm 829$ vs. $952 \pm 564 \mathrm{~mL})$, while the femoral component revision had a significantly more total blood loss compared with the acetabular component revision $(1,078 \pm 829$ vs. $690 \pm 479 \mathrm{~mL}, \mathrm{P}<0.05)$.

To minimize the interference of the heterogeneity of subtypes of revision THA, we evaluated the efficacy and safety of TXA in a revision subgroup. To our knowledge, this was the first study to evaluate the efficacy and safety of both intravenous TXA and combined TXA and to compare the two protocols in a subtype of revision THA.

Our results showed both intravenous TXA and combined TXA reduced calculated blood loss and transfusion volume without increasing the risk of VTE, which was consistent with findings from previous studies concerning intravenous TXA use in revision THA $(7,8,11,12)$. Specifically, Peck $e t$ al. (8) evaluated the efficacy of intravenous TXA on four subtypes of revision THA. Compared with the control group, intravenous TXA reduced the estimated blood loss (845.2 vs. $1,095 \mathrm{~mL}, \mathrm{P}<0.001$ ), intraoperative transfusion volume (0.93 vs. 1.37 units, $\mathrm{P}<0.05)$, and total transfusion volume (1.79 vs. 3.33 units, $\mathrm{P}<0.001)$ in revision THA involving deep infection, $\mathrm{PFF}$, and both femoral and acetabular components.

We also found intraoperative transfusion rate was significantly reduced in the combined group, while the postoperative transfusion rate and overall transfusion rate were statistically equivalent to the control group. Hines et al. (9) retrospectively reviewed 3,264 revision THA and found intravenous TXA ( $1 \mathrm{~g}$ at incision plus $1 \mathrm{~g}$ at closure) significantly reduced the transfusion rate in both aseptic revision and septic revision compared with the control group ( $18 \%$ vs. $49 \%, \mathrm{P}<0.001 ; 53 \%$ vs. $73 \%, \mathrm{P}=0.04$; respectively). In contrast, Reichel et al. (7) conducted a prospective cohort study to evaluate an intravenous TXA protocol $(10 \mathrm{mg} / \mathrm{kg}$ preoperatively plus $1 \mathrm{mg} / \mathrm{kg} / \mathrm{h}$ intraoperatively) in revision THA, and results showed that the transfusion rate was similar between the intravenous TXA group and the control group (50\% vs. $65 \%, \mathrm{P}=0.26)$. The discrepancies may result from the heterogeneity of revision procedures or TXA administration protocols. In the present study, a lower perioperative transfusion rate was observed in both the intravenous group and the combined group.

When comparing the intravenous TXA protocol with the combined TXA protocol, we found no significant differences in calculated blood loss, transfusion volume, and transfusion rate with a similar risk of VTE. Wu et al. (13) compared the efficacy and safety between combined TXA $(15 \mathrm{mg} / \mathrm{kg}$ intravenously 10 minutes before incision plus $3 \mathrm{~g}$ 
topically in three stages) and intravenous TXA $(15 \mathrm{mg} / \mathrm{kg}$ intravenously 10 minutes before incision), and results showed the combined TXA group had a significantly less total blood loss and significantly lower transfusion rate. Although there was a significant difference in total blood loss among the revision of both components, isolated femoral revision, and isolated acetabular revision, subgroup analysis of TXA was not conducted. In the present study, a pairwise comparison showed calculated blood loss, transfusion volume, and transfusion rate were nonsignificant higher in the intravenous group compared with the combined group, implying underlying benefits of the combined TXA protocol.

There were several limitations of this study. First, four senior surgeons with variable numbers of revision cases were involved in revision procedures from January 2010 to December 2018. Although all of the surgeons performed revision procedures through a posterolateral approach, the heterogeneity of surgical skills still was present, and surgical techniques got improved over time, which may have caused bias in this study. Second, Doppler vascular ultrasound scan or pulmonary vascular CT scan was not routinely recommended to rule out postoperative VTE in patients without relevant clinical manifestations, which may cause undiagnosed VTE events. We merely included VTE events occurring in hospital, and long-term follow-up of discharged patients is required for further evaluation of the safety of TXA. Finally, there was a limited population in this retrospective study. Randomized clinical trials with a larger population are required to fully evaluate the efficacy and safety of TXA in subtypes of revision THA.

\section{Conclusions}

For unilateral major revision THA, both intravenous TXA and combined TXA effectively reduced calculated blood loss and perioperative transfusion volume with comparable perioperative transfusion rate and the risk of VTE. More researches are required to explore the optimal TXA administration protocol in subtypes of revision THA.

\section{Acknowledgments}

Funding: None.

\section{Footnote}

Reporting Checklist: The authors have completed the
STROBE reporting checklist. Available at http://dx.doi. org/10.21037/apm-19-372

Data Sharing Statement: Available at http://dx.doi. org/10.21037/apm-19-372

Conflicts of Interest: All authors have completed the ICMJE uniform disclosure form (available at http://dx.doi. org/10.21037/apm-19-372). The authors have no conflicts of interest to declare.

Ethical Statement: The authors are accountable for all aspects of the work in ensuring that questions related to the accuracy or integrity of any part of the work are appropriately investigated and resolved. This study was conducted in accordance with the Declaration of Helsinki (as revised in 2013) and was approved by the Ethics Committee of the Second Xiangya Hospital of Central South University (2019-192). Informed consent was waived because of the nature of a retrospective study. The patient's personal data have been secured.

Open Access Statement: This is an Open Access article distributed in accordance with the Creative Commons Attribution-NonCommercial-NoDerivs 4.0 International License (CC BY-NC-ND 4.0), which permits the noncommercial replication and distribution of the article with the strict proviso that no changes or edits are made and the original work is properly cited (including links to both the formal publication through the relevant DOI and the license). See: https://creativecommons.org/licenses/by-nc-nd/4.0/.

\section{References}

1. Xie J, Zhang S, Chen G, et al. Optimal route for administering tranexamic acid in primary unilateral total hip arthroplasty: results from a multicenter cohort study. Br J Clin Pharmacol 2019;85:2089-97.

2. Wang D, Wang HY, Luo ZY, et al. Finding the optimal regimen for oral tranexamic acid administration in primary total hip arthroplasty: a randomized controlled trial. J Bone Joint Surg Am 2019;101:438-45.

3. Wang D, Yang Y, He C, et al. Effect of multiple doses of oral tranexamic acid on haemostasis and inflammatory reaction in total hip arthroplasty: a randomized controlled trial. Thromb Haemost 2019;119:92-103.

4. Clavé A, Gérard R, Lacroix J, et al. A randomized, doubleblind, placebo-controlled trial on the efficacy of tranexamic 
acid combined with rivaroxaban thromboprophylaxis in reducing blood loss after primary cementless total hip arthroplasty. Bone Joint J 2019;101-B:207-12.

5. Free MD, Owen DH, Pascoe E, et al. Transfusion rates with intravenous tranexamic acid in total hip arthroplasty performed using the direct anterior approach. Hip Int 2019;29:511-5.

6. Fillingham YA, Ramkumar DB, Jevsevar DS, et al. The efficacy of tranexamic acid in total hip arthroplasty: a network meta-analysis. J Arthroplasty 2018;33:3083-9.e4.

7. Reichel F, Peter C, Ewerbeck V, et al. Reducing blood loss in revision total hip and knee arthroplasty: tranexamic acid is effective in aseptic revisions and in second-stage reimplantations for periprosthetic infection. Biomed Res Int 2018;2018:3891870.

8. Peck J, Kepecs DM, Mei B, et al. The effect of preoperative administration of intravenous tranexamic acid during revision hip arthroplasty: a retrospective study. J Bone Joint Surg Am 2018;100:1509-16.

9. Hines JT, Hernandez NM, Amundson AW, et al. Intravenous tranexamic acid safely and effectively reduces transfusion rates in revision total hip arthroplasty. Bone Joint J 2019;101-B:104-9.

10. Goel R, Buckley P, Sterbis E, et al. Patients with infected

Cite this article as: Mei L, Li H, Zhu W, Luo Y, Mao X. Efficacy and safety of tranexamic acid in unilateral major revision total hip arthroplasty. Ann Palliat Med 2020;9(5):2466-2473. doi: 10.21037/apm-19-372 total hip arthroplasty undergoing 2-stage exchange arthroplasty experience massive blood loss. J Arthroplasty 2018;33:3547-50.

11. Park KJ, Couch CG, Edwards PK, et al. Tranexamic acid reduces blood transfusions in revision total hip arthroplasty. J Arthroplasty 2016;31:2850-5.e1.

12. Mariani P, Buttaro MA, Slullitel PA, et al. Transfusion rate using intravenous tranexamic acid in hip revision surgery. Hip Int 2018;28:194-9.

13. Wu YG, Zeng Y, Yang TM, et al. The efficacy and safety of combination of intravenous and topical tranexamic acid in revision hip arthroplasty: a randomized, controlled trial. J Arthroplasty 2016;31:2548-53.

14. Garvin KL, Feschuk CA, Sekundiak TD, et al. Blood salvage and allogenic transfusion needs in revision hip arthroplasty. Clin Orthop Relat Res 2005;441:205-9.

15. Zarin J, Grosvenor D, Schurman D, et al. Efficacy of intraoperative blood collection and reinfusion in revision total hip arthroplasty. J Bone Joint Surg Am $2003 ; 85: 2147-51$.

16. Huerfano E, Huerfano M, Shanaghan KA, et al. Topical tranexamic acid in revision total knee arthroplasty reduces transfusion rates and may be associated with earlier recovery. J Arthroplasty 2019;34:S249-55. 